\title{
El papel de las actitudes lingüísticas del alumnado de E.S.O. en el rendimiento y motivación hacia el estudio de una lengua
}

\author{
Arantzazu Rodríguez-Fernández, Universidad del País Vasco/Euskal \\ Herriko Unibertsitatea, País Vasco, España \\ Urtzi Ruiz-de-Azua-Ormaza, UPV/EHU, País Vasco, España \\ Inge Axpe, Universidad del País Vasco/Euskal Herriko Unibertsitatea \\ (UPV/EHU), País Vasco, España
}

\begin{abstract}
Resumen: Aunque la mayor parte de los autores está de acuerdo en que las actitudes están formadas por tres componentes referidos a lo cognitivo, lo afectivo y lo conductual, y que gran cantidad de estudios han examinado las actitudes lingüísticas en función de variables sociopersonales y psicológicas como la motivación, son muy escasos los trabajos que han estudiado la relación entre ambas desde el aprendizaje de una segunda lengua. Participaron en esta investigación un total de 1356 estudiantes de Educación Secundaria Obligatoria (676 hombres y 680 mujeres), que respondieron tanto a una adaptación del Cuestionario de Estrategias de Aprendizaje y Motivación (CEAMII) de Roces, Tourón y González (1995) para valorar la motivación escolar, como al Cuestionario de Actitudes Lingüísticas (CAL) de Ruiz de Azúa-Ormaza (2012), destinado a medir las actitudes lingüisticas. Además, se obtuvieron las calificaciones escolares relativas a la última evaluación de los participantes. Los resultados indican que las calificaciones retroalimentan la relación entre las actitudes y la motivación hacia el estudio del idioma de forma que cuanto mejores sean las notas del alumno estará más motivado hacia el aprendizaje de dicha materia y las actitudes hacia la misma serán más positivas que al contrario. Asimismo, se comprueba que la motivación para el aprendizaje de una lengua posee una capacidad de predicción sobre las actitudes hacia esta lengua mayor que a la inversa.
\end{abstract}

Palabras Clave: Actitudes Lingüísticas, Rendimiento Académico, Motivación, Estudio, Lengua

Abstract: Most of the authors agree that attitudes are formed by three components, cognitive, affective and behavioural. In addition, a large number of studies have examined the attitudes of linguistic function in personal-social and psychological variables such as motivation. Nevertheless, very few studies have examined the relationship between the two variables (motivation and linguistic attitudes) from a second language learning. An adaptation of the Roces, Tourón and González's Motivated Strategies for Learning Questionnaire (1995) along with the Ruiz de Azúa-Ormaza's Linguistic Attitudes Questionnaire (2012) were administered to a sample of 1356 High School students (676 Men and 680 women). Latest school grades were also included. The results show that grades offer feedback on the relationship between the attitudes and motivation toward the study of the language, in such a way that the better the students' academic marks are, the higher the motivation and the more positive the attitudes toward the subject's learning. It has also been found that motivation for language learning has a higher predictive capacity on the attitudes toward this language than vice versa.

Keywords: Linguistic Attitudes, Academic Grades, Motivation, Study, Language

Revista Internacional de Educación y Aprendizaje

Volumen 1, 2013, http://sobrelaeducacion.com/revistas/coleccion/, ISSN 2255-453X

(C) Global Knowledge Academics. Arantzazu Rodríguez-Fernández et al.

Todos los Derechos Reservados. Permisos: soporte@gkacademics.com

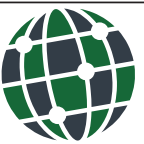




\section{Introducción}

\section{Las actitudes lingüísticas}

ON MUCHAS LAS aportaciones teóricas que se han hecho sobre las actitudes, siendo una de las más interesantes la "teoría de la acción razonada" de Fishbein y Ajzen (1975), en la cual se describe que una coincidencia sumativa o combinación de las actitudes y la norma subjetiva (originada por creencias normativas), que a su vez representa la presión del medio sobre el individuo, da lugar a una intención conductual que es lo más próximo a la conducta real del individuo.

Todo esto lleva a pensar que las actitudes son un constructo interno de cada individuo. Así, podríamos definir la actitud como "un sentimiento negativo o positivo (o incluso neutro o de indiferencia) hacia una persona, objeto o cuestión que ha sido conocida por medio de la interacción social" (Lasagabaster, 2003; p. 535).

Pero más que una definición, lo que muchos autores reflejan en sus trabajos sobre este constructo son los elementos que lo constituyen. Según diferentes autores (Fernández Paz, 2001; Lasagabaster, 2003), las actitudes están constituidas por tres elementos: el cognitivo, conjunto de conceptos y creencias que formarían la base y que dan una apariencia de racionalidad; el afectivo, formado por las emociones, sentimientos, vivencias propias, que hacen que sea el componente esencial de las actitudes, dado que garantizan su persistencia y las hace inmunes a los razonamientos que se les podrían oponer; y por último, el conductual que más que un elemento interno de las actitudes sería la expresión práctica y observable de éstas, que nos permite de alguna manera identificarlas.

En el estudio de las actitudes lingüísticas la mayoría de los autores utilizan una perspectiva mentalista, la cual considera que las actitudes son un estado mental interno de cada individuo, que tiene como respuesta diferentes formas de comportamiento. Pero esta perspectiva plantea un problema de investigación: que los estados mentales internos, al no ser observables, deben ser inferidos por el propio investigador a través del comportamiento del sujeto observado, o bien debe ser aportado por el propio sujeto a través de medidas autoinformadas (Appel y Muysken, 1986).

Las actitudes están determinadas en gran parte por las creencias del sujeto y por lo tanto son privadas, en cambio las conductas son públicas y las conductas reales son aquellas que han sido con frecuencia sometidas a las presiones sociales, es decir, a menudo no podemos asemejar las actitudes a conductas, o ni siquiera a opiniones a causa del condicionamiento de esa presión social (Huguet y Madariaga, 2005).

A la hora de hablar de actitudes lingüísticas, no podemos pasar por alto la relación entre lengua e identidad, ya que la lengua no sólo es utilizada como instrumento para la comunicación, sino también para la transmisión de significados o connotaciones sociales, y cuando una lengua tiene un significado social, será evaluada en relación al status social de sus usuarios. Por tanto, las actitudes lingüísticas serán actitudes sociales (Appel y Muysken, 1986) lo que hace que grupos sociales o étnicos adopten unas actitudes como las prácticas culturales, la forma de vestir, la lengua que hablan... en referencia a los otros, y esto les defina como grupo. 


\section{Las actitudes lingüísticas hacia el euskera, la motivación y el rendimiento académico}

Son numerosos los estudios que se han realizado con el objetivo de esclarecer la relación entre actitudes, motivación y competencia lingüística en sujetos dedicados al aprendizaje de una segunda lengua. Y aunque la mayoría de los estudios reflejan una clara relación entre dichos constructos, el debate se centra en la determinación de la secuencia causa/efecto de esta relación.

En este sentido, algunos autores señalan que la motivación para el aprendizaje de una segunda lengua determinará su grado de competencia lingüística, y a su vez algunos autores afirman que existe una relación entre el rendimiento en el aprendizaje de una lengua y las actitudes de esa persona hacia los sujetos que tienen esa lengua como lengua materna. Lo cual refuerza la teoría de que las actitudes no se heredan, sino que se obtienen en los procesos de socialización, por lo que cada individuo adquirirá sus propias actitudes en las diferentes interacciones (Baker, 1997). Ahora bien, no parece quedar claro qué influencia tiene la motivación sobre las actitudes hacia el propio aprendizaje de esa lengua.

Por el contrario, otros autores mantienen que las actitudes son características propias del individuo, relativamente estables y, por lo tanto, influirán y determinarán el proceso de aprendizaje de una segunda lengua y no al revés (Sánchez y Rodríguez, 1997).

Pero es un tercer enfoque el que en los últimos años está tomando mayor consistencia. Éste postula que probablemente estos dos constructos están determinados entre sí y ambos son causa y efecto de igual manera (Huguet, 2005). De ello podríamos deducir que las actitudes son en parte heredadas y relativamente estables, pero también modificables gracias a las diferentes interacciones en el proceso de socialización del individuo.

A pesar de que la cantidad de trabajos que de uno u otro modo estudian las actitudes lingüísticas es amplia, muchos se centran en el análisis de las propias actitudes en función de diferentes variables sociopersonales tales como la edad, lugar de residencia, país de procedencia, sociedades con contacto de lenguas... (Fernández Paz, 2001; Huguet y Madariaga, 2005; Lasagabaster, 2003; Mar-Molinero, 2001; Oroz, 1998; Solís, 2001) y algunos otros las relacionan con la motivación (Baker, 1997; Sánchez y Rodríguez de Tembleque, 1997). Pero son escasos los que se han centrado en el estudio de éstas hacia una asignatura relativa al aprendizaje de una segunda lengua minoritaria, e incluso se deja de lado el análisis de las relaciones que dichas actitudes puedan tener con el rendimiento académico en aquella. Por tanto, en el presente trabajo se pretende examinar la relación existente entre las actitudes lingüísticas hacia el estudio de una lengua minoritaria (el euskera) para con la motivación escolar y el rendimiento académico en dicha asignatura.

Así mismo, tampoco existe un acuerdo entre los autores sobre cuál pudiera ser la dirección causa/efecto entre las actitudes lingüísticas y la motivación (Baker, 1997; Sánchez y Rodríguez, 1997; Huguet, 2005). Tema primordial de tratar, si desde las aulas queremos incidir sobre aquellos aspectos que permitan mejorar el rendimiento de nuestros alumnos en el aprendizaje del euskera. 


\section{Método}

\section{Participantes}

Participan en este estudio un total de 1356 estudiantes de Educación Secundaria Obligatoria repartidos de forma muy equitativa entre los dos sexos, puesto que $676(49,9 \%)$ son hombres y $680(50,1 \%)$ son mujeres, con edades comprendidas entre los 10 y los 16 años de edad. Atendiendo al modelo lingüístico en el que cursan la ESO, se les dividió en tres grupos: modelo A con un tota de 185 participantes (13,6\%); modelo B compuesto por 285 participantes (21\%); y modelo D, con un total de $886(65,2 \%)$. Todos los participantes eran estudiantes en colegios de Álava, Vizcaya y Guipuzcoa.

Aunque este número de participantes conforma la muestra final, el total de participantes inicial era superior, concretamente de 1455 . No obstante, debido a que algunos dejaron sin contestar un porcentaje de ítems superior al 10\% del total que conformaban los cuestionarios, se decidió prescindir de ellos (un total de 65). Asimismo, otra condición de eliminación fueron las respuestas no sinceras, detectadas a partir de dos ítems de sinceridad (Nunca he visto una bicicleta y Este mes he cruzado mas de 5 veces el océano) contenidos en uno de los cuestionarios aplicados, eliminando de esta forma a 17 participantes. También se suprimieron de la muestra 17 alumnos por estar exentos de la asignatura de euskera, ya que no eran validos para el objetivo del presente trabajo, pues resulta esencial correlacionar diferentes variables con los resultados académicos en dicha materia.

\section{Procedimiento}

Para la elección de la muestra se optó por el método de la selección al azar estratificada, es decir, se escogieron varios centros educativos al azar y dentro de esos centros, se volvieron a seleccionar varias clases. Tras ponerse en contacto con los diversos centros, se pidió permiso tanto a los directores de los mismos como a los padres de los alumnos, en caso de que estos fuesen menores de edad.

A fin de controlar las diferentes amenazas que pudieran sesgar los resultados obtenidos (amenaza de pruebas, reactividad a la prueba experimental, deseabilidad social, etc.) se llevaron a cabo distintos controles de las mismas tales como la equiponderación intragrupo aleatoria (administrar diferentes secuencias de tratamiento a distintos subgrupos de sujetos escogidos al azar), desarrollo de una metodología de ciego único (evitar que los participantes conozcan la finalidad de la investigación) y asegurar a los sujetos tanto el anonimato de las respuestas como la total voluntariedad de la participación en la investigación.

Fueron los autores de este trabajo los encargados de aplicar los instrumentos de medida, a los que los participantes respondieron de forma individual en un período de tiempo que oscilaba entre los 20 y los 40 minutos. Asimismo, fueron los encargados de atender individualmente las dudas que fueron surgiendo a los participantes durante su cumplimentación.

Una vez recogidas todas las respuestas y tras su inclusión en una base de datos, se procede al análisis estadístico de los datos con el programa SPSS 18.0 para Windows. Para comprobar el grado de relación entre las tres variables cuantitativas, se seleccionó la prueba de correlaciones de Pearson (1896) de orden cero con un nivel crítico bilateral, y la exclusión de casos según pareja, a fin de eliminar de los cálculos del coeficiente de correlación los casos con algún valor perdido en alguna de las dos variables que se fueran a correlacionar, si los hubiera. 
El grado en el que el rendimiento en el idioma vasco pudiera estar mediando la relación entre las actitudes hacia dicho idioma y la motivación para su estudio, fue analizado a partir de la prueba de correlaciones parciales y también con exclusión de casos según pareja. Finalmente, otra prueba empleada en los análisis estadísticos es la regresión lineal. Ésta mide la posible capacidad predictiva o de incidencia de una serie de variables independientes sobre una única dependiente, proporcionando el grado de influencia de cada una de ellas sobre ésta última. Asimismo, ofrece el dato del grado de correlación entre el conjunto de variables.

\section{Variables e instrumentos de medida}

Para medir la motivación se empleó la traducción del MSLQ (Pintrich, 1991) al castellano, denominada Cuestionario de Estrategias de Aprendizaje y Motivación (CEAM II) de Roces, Tourón y González (1995), con una fiabilidad de conjunto de la escala alta $(\alpha=.82)$. Ahora bien, de las seis escalas que componen la parte del cuestionario relativa al aspecto motivacional con un total de 31 ítems, únicamente se han tenido en cuenta las seis escalas (Orientación extrínseca, Orientación intrínseca, Valoración de la tarea, Autoeficacia, Creencias de control y Ansiedad) para el modelo de regresión. En el resto de análisis se tuvieron en cuenta exclusivamente las escalas que evalúan las metas de orientación intrínseca (o también llamada motivación intrínseca porque aluden al grado en que los sujetos realizan las tareas y acciones por el interés que les genera la actividad misma, considerándola como un fin en sí misma y no como un medio para alcanzar otras metas) y las metas de orientación extrínseca (o motivación extrínseca, que se refieren al grado en que los individuos realizan una determinada acción con el objetivo de satisfacer otros motivos no relacionados con la actividad en sí misma, sino con la consecución de otras metas, como obtener buenas notas, lograr reconocimiento por parte de los demás, evitar el fracaso, etc.), si bien en este caso haciendo referencia al estudio hacia la asignatura de euskera. La razón de esta selección reside en que las investigaciones llevadas a cabo sobre el tema establecen estos dos tipos de orientaciones entendidas como los dos dominios que englobarían el resto de componentes (Alonso, 2005; González, 2005; de Caso et al., 2010). A todos los ítems se responde con arreglo a una escala de tipo Likert de 7 puntos, donde 1 = nunca y 7 siempre. A continuación se describen las 6 escalas empleadas:

A la hora de evaluar las actitudes lingüísticas hacia el estudio de un idioma se utilizó el Cuestio nario de Actitudes Lingüísticas -CAL- de Ruiz de Azúa, (2012) explicitando en los ítems que se referían todos ellos hacia la lengua vasca. El planteamiento subyacente a dicho cuestionario se encuentra en la teoría de las actitudes, según la cual éstas se componen de tres factores que se corresponden con cada una de las escalas del CAL: a) el Componente cognitivo, que hace referencia al conjunto de conceptos y creencias sobre el euskera; b) el Componente afectivo, formado por las emociones, sentimientos y vivencias propias referidas al euskera; y c) el Componente conductual que se corresponde con el conjunto de expresiones prácticas, observables y medibles relativas al euskera. La fiabilidad para el conjunto de la escala es excelente con un alfa de Cronbach $=.94$.

Por último, el rendimiento en el aprendizaje del idioma vasco se midió en una escala de 0 a 10 a partir de la última calificación escolar en dicha asignatura, más próxima en fecha al día de la aplicación de la batería de cuestionarios comentada previamente. Tales calificaciones fueron proporcionadas por el profesorado del alumnado al que se le administró el conjunto de cuestionarios. 


\section{Resultados}

Uno de los objetivos del presente estudio es el análisis de la relación entre las actitudes lingüísticas, la motivación y el rendimiento en el aprendizaje del idioma vasco, de ahí que el primer paso sea examinar el grado de relación del resultado académico en el vasco, con respecto a los tres componentes de las actitudes lingüísticas (conducta, afecto y cognición), a partir del método de correlación de Pearson (cf. Tabla 1).

Tabla 1: Correlaciones entre las actitudes y el rendimiento

\begin{tabular}{|c|c|c|}
\hline & & EUSKERA \\
\hline \multirow{2}{*}{ CONDUCTA } & Correlación de Pearson &, $275(* *)$ \\
\hline & Sig. (bilateral) &, 000 \\
\hline \multirow{2}{*}{ AFECTO } & Correlación de Pearson &, $281(* *)$ \\
\hline & Sig. (bilateral) &, 000 \\
\hline \multirow{2}{*}{ COGNICIÓN } & Correlación de Pearson &, $231(* *)$ \\
\hline & Sig. (bilateral) &, 000 \\
\hline \multirow{2}{*}{ EUSKERA } & Correlación de Pearson & 1 \\
\hline & Sig. (bilateral) & \\
\hline
\end{tabular}

Tal como se puede observar en la tabla, el rendimiento en la asignatura de euskera correlaciona de forma positiva y significativa con los tres componentes de la actitudes lingüísticas, pero lo hace especialmente con el componente afectivo $(r=.281 ; p<.001)$ y con el componente conductual $(r=.275 ; p<.001)$, siendo algo menor la relación que presenta con el componente cognitivo $(r=.231 ; p<.001)$. Dicho de otro modo, cuanto más positivo sea el afecto que un estudiante de Educación Secundaria Obligatoria muestra hacia el euskera, mayor cantidad de conductas mantenga relacionadas con el idioma (tales como leer o escuchar la radio en ese idioma, utilizar el idioma de forma habitual en su casa, con sus amigos, con sus compañeros de estudio, etc.), y cuanta mejor consideración tenga por tal idioma, mayores resultados académicos obtendrá en el aprendizaje del mismo.

Una vez analizada la relación entre las actitudes y el rendimiento en el estudio del idioma, queda por determinar si dicho rendimiento tiene algún tipo de efecto sobre la relación entre los tres componentes de las actitudes lingüísticas y los dos tipos de motivación (extrínseca e intrínseca). Para ello, el primer paso consiste en ver la relación estricta (de orden cero) entre ambas variables, obviando el papel que pueda ejercer el rendimiento académico. Tales resultados se exponen en la tabla 2 . 
Tabla 2: Correlaciones de orden cero entre las actitudes y la motivación

\begin{tabular}{|c|c|c|c|c|c|c|}
\hline & & $\begin{array}{l}\text { CON- } \\
\text { DUCTA }\end{array}$ & AFECTO & $\begin{array}{c}\text { COGNI- } \\
\text { CIÓN }\end{array}$ & $\begin{array}{l}\text { M. } \\
\text { INTRÍN- } \\
\text { SECA }\end{array}$ & $\begin{array}{l}\text { M. EX- } \\
\text { TRÍN- } \\
\text { SECA }\end{array}$ \\
\hline CON- & $\begin{array}{l}\text { Correlación de } \\
\text { Pearson }\end{array}$ & 1 &, $672(* *)$ &, $578(* *)$ &, $211(* *)$ & ,034 \\
\hline & Sig. (bilateral) & . & ,000 & ,000 & ,000 & ,213 \\
\hline AFECTO & $\begin{array}{l}\text { Correlación de } \\
\text { Pearson }\end{array}$ & & 1 &, $793(* *)$ &, $257(* *)$ &, $055(*)$ \\
\hline & Sig. (bilateral) & & & , 000 & ,000 & ,042 \\
\hline COGNI- & $\begin{array}{l}\text { Correlación de } \\
\text { Pearson }\end{array}$ & & & 1 &, $226(* *)$ &, 050 \\
\hline & Sig. (bilateral) & & & . & ,000 & ,068 \\
\hline M. INTRÍN- & $\begin{array}{l}\text { Correlación de } \\
\text { Pearson }\end{array}$ & & & & 1 &, $369(* *)$ \\
\hline & Sig. (bilateral) & & & & . & , 000 \\
\hline M. EXTRÍN- & $\begin{array}{l}\text { Correlación de } \\
\text { Pearson }\end{array}$ & & & & & 1 \\
\hline & Sig. (bilateral) & & & & & . \\
\hline
\end{tabular}

Los resultados indican que existe una relación significativa y de carácter positivo entre las actitudes lingüísticas y la motivación intrínseca, aunque de carácter muy moderado. Así, la conducta presenta un grado de relación de Pearson $r=.211(\mathrm{p}<.001)$; ligeramente más elevada es la que presenta la cognición $(r=.226 ; \mathrm{p}<.001)$ y las más elevada de todas se da para con el componente afectivo $(r=.257 ; \mathrm{p}<.001)$. No sucede lo mismo con la motivación extrínseca, pues no parece mantener ningún tipo de interdependencia para con las actitudes (componente conductual: $r=.034 ; \mathrm{p}>.05$; componente cognitivo: $r=.050 ; \mathrm{p}>.05$ ), salvo con su componente afectivo con el que guarda un grado de correlación muy débil $(r=.055 ; \mathrm{p}<.05)$. Por tanto, se puede concluir que aquellos alumnos y alumnas con mejores actitudes hacia el idioma vasco, también presentan una mayor motivación intrínseca por el estudio de dicha lengua, no así una mayor motivación extrínseca, de cuya relación no se han hallado pruebas empíricas.

Tras el exploración de las correlaciones de orden cero, el segundo paso es extraer las correlaciones parciales entendiendo como variables últimas de la correlación las actitudes lingüísticas y la motivación extrínseca e intrínseca, y como variable mediadora de la relación entre ambas estaría el rendimiento académico en la lengua vasca del alumnado de Educación Secundaria Obligatoria (cf. tabla 3). 
Tabla 3: Correlaciones parciales entre las actitudes y la motivación, controlando para el rendimiento escolar

\begin{tabular}{|l|l|l|l|l|l|l|}
\hline \multicolumn{2}{|c|}{} & $\begin{array}{l}\text { CON- } \\
\text { DUCTA }\end{array}$ & AFECTO & $\begin{array}{l}\text { COGNI- } \\
\text { CIÓN }\end{array}$ & $\begin{array}{l}\text { M. } \\
\text { INTRÍN- } \\
\text { SECA }\end{array}$ & $\begin{array}{l}\text { M. } \\
\text { EXTRÍN- } \\
\text { SECA }\end{array}$ \\
\hline \multirow{2}{*}{$\begin{array}{l}\text { CON- } \\
\text { DUCTA }\end{array}$} & $\begin{array}{l}\text { Correlación de } \\
\text { Pearson }\end{array}$ & 1 &, 644 &, 5501 &, $150(* *)$ &, 0128 \\
\cline { 2 - 7 } & Sig. (bilateral) & &, 000 &, 000 &, 000 &, 638 \\
\hline \multirow{2}{*}{ AFECTO } & $\begin{array}{l}\text { Correlación de } \\
\text { Pearson }\end{array}$ & & 1 &, $7793(* *)$ &, $198(* *)$ &, $0347\left(^{*}\right)$ \\
\cline { 2 - 7 } & Sig. (bilateral) & &. &, 000 &, 000 &, 202 \\
\hline \multirow{2}{*}{$\begin{array}{l}\text { COGNI- } \\
\text { CIÓN }\end{array}$} & $\begin{array}{l}\text { Correlación de } \\
\text { Pearson }\end{array}$ & & & 1 &, $176(* *)$ &, 032 \\
\cline { 2 - 7 } & Sig. (bilateral) & & &. &, 000 &, 232 \\
\hline \multirow{2}{*}{$\begin{array}{l}\text { M. INTRÍN- } \\
\text { SECA }\end{array}$} & $\begin{array}{l}\text { Correlación de } \\
\text { Pearson }\end{array}$ & & & & 1 &, $362(* *)$ \\
\cline { 2 - 7 } & Sig. (bilateral) & & & &. &, 000 \\
\hline \multirow{2}{*}{$\begin{array}{l}\text { M. EXTRÍN } \\
\text { SECA }\end{array}$} & $\begin{array}{l}\text { Correlación de } \\
\text { Pearson }\end{array}$ & & & & & 1 \\
\cline { 2 - 7 } & Sig. (bilateral) & & & & &. \\
\hline
\end{tabular}

Se puede observar que, efectivamente, el rendimiento académico se puede considerar como variable moderadora entre las actitudes lingüísticas y la motivación, puesto que al controlar el efecto que ejerce en el análisis de correlación de Pearson, los niveles de relación entre ambas bajan sensiblemente: el grado de relación entre la motivación intrínseca y la conducta desciende de $\mathrm{r}=.211(\mathrm{p}<.001)$ a $\mathrm{r}=.150(\mathrm{p}<.001)$, la correlación entre dicha motivación y el componente afectivo de las actitudes disminuye de $r=.257(\mathrm{p}<.001)$ a $r=.198(\mathrm{p}<-.001)$ y el componente congitivo desciende de $r=.226(\mathrm{p}<.001)$ a $r=.176(p<.001)$, manteniéndose la interdependencia positiva siempre significativa en los tres casos.

En otras palabras, el rendimiento académico en el estudio de la lengua vasca tiene un efecto medio de .056 sobre la relación que se viene estudiando; y más concretamente, de .061 sobre la relación entre la motivación intrínseca y las conductas de uso de la lengua vasca, de .059 para con los sentimientos más positivos hacia dicho idioma, y de .050 con respecto al componente cognitivo, de tal forma que si se controla el rendimiento académico en euskera, el grado de relación entre las actitudes lingüísticas y la motivación intrínseca desciende significativamente.

Por último, queda por averiguar cuál pudiera ser la dirección causa/efecto entre las actitudes lingüísticas y la motivación, y para ello se sometieron a prueba dos modelos de regresión, cuyos resultados se pueden ver en la tabla 4: uno en el que se consideran como variable predictiva las actitudes sobre la motivación como variable dependiente, y el otro en el que se hace a la inversa, considerando la motivación como variable predictiva y las actitudes como variable dependiente, si bien en esta caso se incluyeron todas las medidas que el CEAM 
facilita sobre la motivación académica (metas de orientación intrínseca y extrínseca, autoeficacia, ansiedad ante la tarea, valor de la tarea y creencias de control).

Tabla 4: Modelos de regresión entre las actitudes y la motivación

\begin{tabular}{|c|c|c|c|c|c|c|}
\hline \multicolumn{7}{|c|}{ Regresión de las actitudes sobre la motivación } \\
\hline VD & & $\mathrm{F}$ & $\mathrm{p}$ & $\operatorname{Beta}(\beta)$ & $\mathrm{t}$ & $\mathrm{p}$ \\
\hline \multirow{4}{*}{ MOTIVACIÓN } & (Constante) & \multirow{4}{*}{48.777} & \multirow{4}{*}{.000} & & 27.851 & $.000 * * *$ \\
\hline & Conducta & & & .193 & 3.064 & $.002 * *$ \\
\hline & Afecto & & & .331 & 4.516 & $.000 * * *$ \\
\hline & Cognición & & & .069 & 0.966 & .335 \\
\hline \multicolumn{7}{|c|}{ Regresión de la motivación sobre las actitudes } \\
\hline VD & & $\mathrm{F}$ & $\mathrm{p}$ & Beta $(\beta)$ & $\mathrm{t}$ & $\mathrm{p}$ \\
\hline \multirow{7}{*}{$\begin{array}{l}\text { ACTITUDES } \\
\text { LINGÜÍSTI- } \\
\text { CAS }\end{array}$} & (Constante) & \multirow{7}{*}{51.658} & \multirow{7}{*}{.000} & & 3.833 & $.000 * * *$ \\
\hline & Autoeficacia & & & .028 & 0.506 & .613 \\
\hline & Ansiedad & & & .120 & 2.756 & $.006^{* *}$ \\
\hline & Valor tarea & & & .635 & 13.320 & $.000 * * *$ \\
\hline & Cr. Contr & & & .135 & 2.940 & $.003 * *$ \\
\hline & Or. Intri & & & -.091 & -1.912 & .057 \\
\hline & Or. Extri & & & -.064 & -1.501 & .134 \\
\hline
\end{tabular}

Los resultados indican que la capacidad predictiva de la motivación sobre las actitudes $\left(\mathrm{R}^{2}=.465\right)$, en contra de lo planteado en la hipótesis, es considerablemente más elevada que a la inversa $\left(\mathrm{R}^{2}=.290\right)$. También, en un análisis más detallado, se puede ver que de los tres componentes de las actitudes, es el afectivo $(\beta=.331 ; \mathrm{p}<.001)$ el que mayor capacidad de influencia presenta sobre la motivación para el aprendizaje del euskera, mientras que no presenta ninguna capacidad de influencia el componente cognitivo $(\beta=.069 ; \mathrm{p}>.05)$. Y, que de los diversos componentes de la motivación medidos, el más influyente sobre las actitudes es la valoración de la tarea $(\beta=.635 ; \mathrm{p}<.001)$, no llegando a presentar ningún tipo de capacidad predictiva ni la autoeficacia ni la orientación extrínseca $(\beta=.028 ; \mathrm{p}>.05$ y $\beta=.064 ; p>.05)$, y sorprendentemente tampoco lo hace la orientación intrínseca $(\beta=-.091$; $\mathrm{p}>.05)$.

\section{Discusión de resultados}

Tras examinar los resultados de esta investigación, queda claro que existe una relación positiva y significativa entre el rendimiento en la asignatura de euskera, los tres componentes de las actitudes lingüísticas (cognitivo, afectivo y conductual) y la motivación hacia el estudio de dicha lengua, de forma que aquellas personas que tienen unas actitudes lingüísticas más positivas son, a su vez, las que obtienen unos resultados académicos más 
elevados en el idioma en cuestión y las que más motivadas hacia el estudio del mismo se encuentra.

Esto hace pensar en la importancia que tiene una educación en la que se incida especialmente en el fomento de actitudes lingüísticas positivas, alentando una conciencia plurilingüe, cierta sensibilidad hacia las lenguas y culturas, y por supuesto el respeto hacia las lenguas y sus hablantes para así poder conseguir no solo unos resultados académicos óptimos en dicha lengua sino también una mejor convivencia entre diferentes culturas. Dicho fomento redundará en una mayor motivación hacia el estudio de la lengua, la cual conllevará a su vez unas actitudes lingüísticas más positivas entrando así en un círculo vicioso cuyos factores continuamente se están retroalimentando teniendo como consecuencia unos resultados académicos mejores en dicho idioma.

Ahora bien, a esta afirmación es necesario hacerle un matiz: no toda motivación académica tienen relación con el rendimiento académico. Repetidamente, los diferentes autores que más a fondo han indagado en el tema de la motivación han llegado a la misma conclusión (Alonso, 2005; González, 2005; de Caso et al., 2010): aunque la motivación pueda estar conformada por distintos componentes, un examen exhaustivo de todos esos componentes o factores revela que se pueden agrupar en dos grandes factores que se corresponden con los dos principales tipos de motivación, a saber, a) la motivación extrínseca (o grado en que los individuos realizan una determinada acción con el objetivo de satisfacer otros motivos no relacionados con la actividad en sí misma, sino con la consecución de otras metas, como obtener buenas notas, lograr reconocimiento por parte de los demás, evitar el fracaso, etc.), y b) la motivación intrínseca (o grado en que los sujetos realizan las tareas y acciones por el interés que les genera la actividad misma, considerándola como un fin en sí misma y no como un medio para alcanzar otras metas). Teniendo en cuenta esta división y los resultados obtenidos en el presente estudio, se puede afirmar, acorde con las pruebas empíricas, que unas mejores actitudes lingüísticas únicamente van a repercutir en la motivación intrínseca, nunca sobre la extrínseca, con las que no parecen presentar ningún tipo de interdependencia.

Por otra parte, se demuestra que la relación entre las actitudes y la motivación intrínseca baja significativamente cuando introducimos las notas como variable moderadora, de forma que se puede concluir que las notas estarían retroalimentando esa relación. Así, cuando las notas son mejores, la persona estaría más motivada hacia el aprendizaje de esa materia y las actitudes hacia el idioma serían más positivos que al contrario. Todo esto podría hacer pensar que la posible base o punto de partida de este entramado de retroalimentaciones sería la motivación intrínseca hacia el estudio de una lengua, la cual incidiría en unas mejores actitudes hacia la misma; ahora bien, cuando en tal relación entran en juego unos mejores resultados escolares en esa lengua, la relación entre ambas variables mejora.

Pero el hecho de que las actitudes lingüísticas también tengan capacidad de incidencia sobre la motivación, la convierten en el constructo en el que con mayor facilidad se podría conseguir una influencia positiva en este entramado de relaciones desde diferentes ámbitos educativos tales como la familia, el colegio o la sociedad en general.

Ahora bien, el trabajo aquí presentado, en el que se analiza el esquema de relaciones entre las actitudes lingüísticas, la motivación y el rendimiento académico en un idioma, no deja de ser un análisis exploratorio de tales relaciones. Desde unpunto de vista metodológico, sería necesario complementarlo con otro tipo de análisis más avanzado y que permita confirmar dicho modelo relacional: la metodología SEM que posibilita la extracción y análisis de modelos de ecuaciones estructurales. 


\section{Referencias}

Alonso Tapia, J. (2005). "Motivaciones, expectativas y valores-intereses relacionados con el aprendizaje: el cuestionario MEVA". Psicothema, 17(3), 404-411.

Appel, R. y Muysken, P. (1986). Bilingüismo y contacto de lenguas. Barcelona: Ariel.

Baker, C. (1997). Fundamentos de educación bilingüe y bilingüismo. Madrid: Cátedra.

De Caso et al. (2010). "Motivación y educación”. En J. I. Navarro y C. Martín (Coords.), Psicología de la Educación para docentes (pp. 133-153). Madrid: Pirámide.

Fernández Paz, A. (2001). "Las actitudes lingüísticas en situaciones de contacto de lenguas: el caso de Galicia”. Textos de Didáctica de la Lengua y de la Literatura, 26, 17-27.

González, A. (2005). Motivación académica. Teoría, aplicación y evaluación. Madrid: Pirámide.

Fishbein, M., y Ajzen, I. (1975). Belief, attitude, intention and b ehaviour. Reading, Mass.: AddisonWesley.

Huguet, A. (2005). "Génesis y desarrollo de las actitudes lingüísticas en contextos bilingües. Análisis de algunas variables del ámbito escolar y sociofamiliar”. Revista de Psicología Social, 20(2), 175-191.

Huguet, A., y Madariaga, J. M. (2005). Fundamentos de la educación bilingüe. Bilbao: Universidad del País Vasco.

Lasagabaster, D. (2003). Trilingüismo en la enseñanza. Actitudes hacia la lengua minoritaria, la mayoritaria y la extranjera. Lleida: Milenio.G

Mar-Molinero, C. (2001). "Identidad nacional y educación bilingüe en el mundo hispano-hablante". Revista de E ducación, 326, 79-97.

Oroz, N. (1998). "Las actitudes hacia la lengua vasca en Navarra". Notas y estudios filológicos, 13, $159-180$.

Pintrich, P. R. (1991). "Editor's comment: Current issues and new directions in motivational theory and research". Educational Psychologist, 26, 199-205.

Roces, C., Tourón, J., y González, M. C. (1995). "Validación preliminar del CEAM II (Cuestionario de Estrategias de Aprendizaje y Motivación II)". Psicológica, 16(3), 347-366.

Ruiz de Azúa-Ormaza, U. (2012). "Motivación, rendimiento académico y actitudes lingüísticas hacia el euskera de alumnado de educación secundaria”. En J. M. Correa y D. Losada (Eds.), Construyendo la identidad investigadora (pp. 37-49). Lejona: Universidad del País Vasco.

Sánchez, M. P., y Rodríguez de Tembleque, R. (1997). El bilingüismo. Bases para la intervención psicológica. Madrid: Síntesis.

Solís, M. (2001). “Actituds per el canvi: educar en la llengua i societat”. Llengua i ús: Revista Técnica de Política L ingüística, 21, 70-73.

\section{Sobre las Autoras}

Dra. Arantzazu Rodríguez-Fernández: Doctora en Psicología por la Universidad del País Vasco, con premio extraordinario obtenido por su tesis doctoral. Desarrolla su actividad investigadora dentro del grupo PSIKOR, reconocido como grupo consolidado por el Gobierno Vasco. Los temas que trabaja son sobre todo el autoconcepto y el bienestar psicológico, si la línea investigadora sobre la que trabaja actualmente es la multidimensionalidad y jerarquía del autoconcepto y su relación con otras variables del ajuste psicosocial como las actitudes o la motivación. 


\section{Urtzi Ruiz-de-Azua-Ormaza:}

Inge Axpe: Inge Axpe es profesora de la Universidad del País Vasco/Euskal Herriko Unibertsitatea (UPV/EHU), en el departamento de Psicología Evolutiva y de la Educación. Participa en el grupo de investigación Psikor. Su principal línea de investigación se desarrolla en relación a la posibilidad de modificar el autoconcepto, especialmente el físico, mediante intervenciones de tipo cognitivo. Igualmente se ha interesado por los estudios de género, especialmente en relación a la posible vinculación entre estereotipos, autoconcepto y sexismo. Actualmente explora también la posible relación del autoconcepto con otras variables implicadas en el ajuste socio-emocional de los sujetos como la resiliencia. 